\title{
Retardo do crescimento em crianças com reação intradérmica positiva para leishmaniose: resultados preliminares
}

\author{
Growth retardation in children with positive Montenegro \\ (leishmanin) test: preliminary results
}

Daniel Ferreira da Cunha, Vítor Carvalho Lara, Jacqueline Pontes Monteiro, Héctor Dardo Romero e Selma Freire de Carvalho da Cunha

\begin{abstract}
Resumo O objetivo deste trabalho foi comparar a ingestão alimentar habitual e a freqüência de retardo do crescimento de crianças com reação intradérmica positiva para leishmaniose (Montenegro-positivas), com um grupo Montenegro-negativo. A ingestão alimentar habitual foi avaliada pelo recordatório de 24 horas e o retardo do crescimento definido segundo critérios da OMS. Crianças Montenegro-positivo $(n=9)$ e Montenegronegativo $(n=17)$ ingeriam, respectivamente, quantidades similares de energia $(1456,8 \pm 314,8 \mathrm{vs} 1316,2 \pm$ $223,8 \mathrm{kcal} / \mathrm{dia})$ e proteínas $(50,4 \pm 16,7 \mathrm{vs} 49,9 \pm 13,9 \mathrm{~g} / \mathrm{dia})$. Déficit de altura foi mais comum em crianças Montenegro-positivas (44,4 vs 5,9). Estes dados sugerem que a infecção prévia pela Leishmania sp afeta desfavoravelmente o estado nutricional de crianças vivendo em área endêmica.
\end{abstract}

Palavras-chaves: Leishmaniose. Estado nutricional. História alimentar.

Abstract The objective of this study was to compare growth retardation frequency, and 24- $h$ food intake data of children with or without positive Montenegro (leishmanin) test, examined in Porteirinha town, Brazil. Daily nutrient intake was determined by 24- $h$ food intake recall and the anthropometric data were compared to the standard values from WHO. Montenegro-positive $(n=9)$ and Montenegro-negative $(n=17)$ groups showed similar age (5.5 \pm 1.9 vs $6.7 \pm 2.3 y)$, and energy $(1,456.8 \pm 314.8$ vs 1,316.2 $\pm 223.8 \mathrm{kcal})$ and protein (50.4 \pm 16.7 vs $49.9 \pm 13.9 \mathrm{~g}$ ) daily consumption, respectively. Montenegro-positive children had higher percentage of stunting than their Montenegro-negative counterparts (44.4 vs 5.9), suggesting that previous Leishmania sp infection had negative impact on children's nutritional status.

Key-words: Leishmaniasis. Nutritional status. Food intake recall.

A alimentação inadequada é uma das causas mais comuns de retardo do crescimento em crianças. Adultos e crianças subnutridos apresentam graus variados de disfunção da imunidade, em especial a mediada por células T, o que os predispõem a infecções oportunísticas, incluindo a leishmaniose visceral (LV) ${ }^{12}$. A LV clássica cursa com quadro de subnutrição grave provocada, entre outros fatores, pela anorexia e pelo aumento do gasto energético e do catabolismo protéico, associados com o aumento de secreção e atividade de interleucinas ${ }^{3}$. São escassos os trabalhos descrevendo o estado nutricional de crianças com sintomatologia inespecífica, indefinida para leishmaniose, subsistindo em áreas com alto risco de reinfecções pela Leishmania. A hipótese do presente estudo é que crianças com reação intradérmica de Montenegro positiva (considerados como tendo sido infectadas por Leishmania $s p$ ) teriam maior prevalência de retardo do crescimento. O objetivo deste trabalho foi comparar a ingestão alimentar habitual e a freqüência de retardo do crescimento de crianças Montenegro-positivas com um grupo de crianças Montenegro-negativas.

Disciplina de Nutrologia do Departamento de Clínica Médica da Faculdade de Medicina do Triângulo Mineiro, Uberaba, MG.

Apoio financeiro: Fundação de Ensino e Pesquisa de Uberaba (FUNEPU).

Endereço para correspondência: Prof. Daniel Ferreira da Cunha. Hospital Escola/FMTM. Av. Getúlio Guaritá 130, 38025-440 Uberaba, MG, Brasil. Telefax: 5534 3318-5335.

e-mail:dfcnutro@mednet.com.br

Recebido para publicação em 22/2/2000. 


\section{MATERIAL E MÉTODOS}

$O$ estudo foi realizado em Porteirinha, MG, região de alta endemicidade para leishmaniose visceral. Foram incluídas todas as crianças (idade entre 3 e 9 anos) que compareceram ao Posto de Saúde durante o mês de julho de 1998, para acompanhamento médico de rotina. Foram registradas as informações das crianças cujos pais concordaram com sua participação no estudo, além de antropometria que incluiu a determinação de peso, altura, circunferência do braço e prega cutânea tricipital, realizados de acordo com Rombeau ${ }^{4}$. O recordatório alimentar de 24 horas $^{6}$ foi obtido das mães das crianças no mesmo dia do atendimento médico, sendo os dados obtidos analisados com o auxílio de um software baseado em banco de dados contendo alimentos consumidos pela população brasileira. Os grupos Montenegro-positivo e Montenegro-negativo foram comparados pelo teste "t" de Student. A freqüência de retardo do crescimento, definida pela relação altura/idade menor que 2 escores " $z$ " em relação ao padrão para crianças normais ${ }^{5}$, foi determinada em cada um dos grupos, e as diferenças de proporção entre os grupos foram comparadas pelo teste exato de Fisher. Diferenças com $p<0,05$ foram consideradas significantes.

\section{RESULTADOS}

Nenhuma das 26 crianças apresentou quadro clínico de leishmaniose, incluindo hepato ou esplenomegalia. Os grupos Montenegro-positivo e Montenegro-negativo apresentaram condições socioeconômicas similares, 0 mesmo ocorrendo com os parâmetros antropométricos (Tabela 1). Documentou-se maior porcentagem de crianças com retardo de crescimento no grupo
Montenegro-positivo (44,4 vs 5,9\%, risco relativo: 7,56 , com intervalo de confiança de 0,99-57,93; $p=0,03$ ). Os dados antropométricos, similares entre os grupos Montenegro-positivos e Montenegro-negativos indicam suficiência do consumo alimentar recente, dado corroborado pela ingestão adequada de energia e proteínas.

Tabela 1 - Antropometria, quantidade de energia e proteínas ingeridas diariamente por crianças com reação intradérmica (Montenegro) positiva ou negativa, estudadas em área endêmica para leishmaniose visceral (Porteirinha, MG).

\begin{tabular}{|c|c|c|c|c|c|c|c|c|c|}
\hline Montenegro & $\begin{array}{l}\text { Retardo do } \\
\text { crescimento }\end{array}$ & Sexo & $\begin{array}{l}\text { Idade } \\
\text { (anos) }\end{array}$ & $\begin{array}{c}\text { Peso } \\
(\mathrm{kg})\end{array}$ & $\begin{array}{l}\text { Altura } \\
(\mathrm{cm})\end{array}$ & $\begin{array}{l}\mathrm{CB} \\
(\mathrm{cm})\end{array}$ & $\begin{array}{l}\text { PCT } \\
(\mathrm{mm})\end{array}$ & $\begin{array}{l}\text { Energia } \\
(\mathrm{kcal} / \mathrm{d})\end{array}$ & $\begin{array}{c}\text { Proteína } \\
(\mathrm{g} / \mathrm{d})\end{array}$ \\
\hline Positivo & $\operatorname{sim}$ & $\mathrm{M}$ & 4 & 12 & 95 & 14,2 & 7,1 & 1009,3 & 31,2 \\
\hline Positivo & $\operatorname{sim}$ & $M$ & 9 & 20 & 123 & 17 & 5,4 & 1512,9 & 44,6 \\
\hline Positivo & $\operatorname{sim}$ & $M$ & 8 & 20 & 120 & 17 & 14,7 & 1589,9 & 66,1 \\
\hline Positivo & $\operatorname{sim}$ & $\mathrm{F}$ & 7 & 16 & 106 & 16 & 7,3 & - & - \\
\hline Positivo & não & $\mathrm{F}$ & 9 & 27 & 130 & 19 & 9,5 & 1231,1 & 51,4 \\
\hline Positivo & não & $\mathrm{F}$ & 5 & 17 & 106 & 17,5 & 12,7 & 1218,9 & 43,7 \\
\hline Positivo & não & $M$ & 3 & 15 & 92 & 16,5 & 7 & 1058,3 & 35,7 \\
\hline Positivo & não & $\mathrm{F}$ & 9 & 30 & 131 & 20 & 13 & 1351,8 & 54,9 \\
\hline Positivo & não & $M$ & 6 & 20 & 117 & 18 & 6,3 & 1557,4 & 70,8 \\
\hline Média \pm desvio & & & 6,7 & 19,7 & 113,3 & 17,2 & 9,2 & 1316,2 & 49,9 \\
\hline padrão & & & 2,3 & 5,7 & 14,3 & 1,7 & 3,4 & 223,8 & 13,9 \\
\hline Negativo & sim & $\mathrm{F}$ & 5 & 14 & 103 & 15,5 & 5,3 & 936,2 & 31,3 \\
\hline Negativo & não & M & 6 & 26 & 131 & 16,5 & 15,3 & 1697,6 & 59,8 \\
\hline Negativo & não & $\mathrm{F}$ & 6 & 19 & 109 & 16 & 6,7 & 1280,2 & 42,8 \\
\hline Negativo & não & M & 8 & 25 & 125 & 18 & 5,8 & 1324,5 & 40,7 \\
\hline Negativo & não & $\mathrm{F}$ & 9 & 25 & 132 & 18 & 7,5 & 1848,5 & 85,2 \\
\hline Negativo & não & $\mathrm{F}$ & 3 & 15 & 95 & 17 & 8,3 & 1285,8 & 36,5 \\
\hline Negativo & não & $M$ & 3 & 17 & 103 & 16 & 5,3 & 1750,9 & 49,1 \\
\hline Negativo & não & $M$ & 5 & 17 & 103 & 16,5 & 5,9 & 1439,6 & 69,4 \\
\hline Negativo & não & $M$ & 7 & 21 & 122 & 19 & 7 & 2044,8 & 66,2 \\
\hline Negativo & não & $\mathrm{F}$ & 5 & 16 & 52 & 17,5 & 12,1 & 1632,4 & 51,9 \\
\hline Negativo & não & $M$ & 3 & 14 & 85 & 16,5 & 9 & 1123,7 & 25,4 \\
\hline Negativo & não & $M$ & 5 & 16 & 108 & 17 & 6 & 1488,7 & 45,8 \\
\hline Negativo & não & $\mathrm{F}$ & 8 & 24 & 118 & 19 & 7,9 & 1877,3 & 76,2 \\
\hline Negativo & não & $M$ & 5 & 18 & 109 & 16 & 4,9 & 1207,3 & 35,7 \\
\hline Negativo & não & $\mathrm{F}$ & 6 & 21 & 119 & 17,5 & 11,5 & 1494,1 & 56,7 \\
\hline Negativo & não & $M$ & 7 & 30 & 131 & 19 & 7,6 & 1310,6 & 50,4 \\
\hline Negativo & não & $M$ & 3 & 14 & 93 & 16,5 & 6,7 & 1022,9 & 33,3 \\
\hline Média \pm desvio & & & 5,5 & 19,5 & 108,1 & 17,1 & 7,8 & 1456,8 & 50,4 \\
\hline padrão & & & 1,9 & 4,9 & 20,1 & 1,1 & 2,8 & 314,8 & 16,7 \\
\hline
\end{tabular}

Obs: A diferença entre os grupos não é significante. 


\section{DISCUSSÃO}

O retardo do crescimento é comum em crianças vivendo em áreas endêmicas para leishmaniose; os resultados deste estudo preliminar sugerem que as crianças Montenegro-positivas constituem o grupo de maior risco nutricional. Limitações metodológicas deste trabalho não permitem inferir se a subnutrição precedeu ou não a infecção pela Leishmania, mas desde que não há evidência clínica de doença em atividade, pode-se especular que a maior porcentagem de retardo do crescimento (stunting) no grupo Montenegropositivo decorreria das repercussões metabólicas da interação hospedeiro-Leishmania, eventualmente associado à repercussão subclínica de retardo do crescimento.

\section{REFERÊNCIAS BIBLIOGRÁFICAS}

1. Cerf BJ, Jones TC, Badaro R, Sampaio D, Teixeira R, Johnson WD. Malnutrition as a risk factor for severe visceral leishmaniasis. The Journal of Infectious Diseases 156:1030-1033, 1987.

2. Harrison LH, Naidu TG, Drew JS, Alencar JE, Pearson RD. Reciprocal relationships between undernutrition and the parasitic disease visceral leishmaniasis. Reviews of Infectious Diseases 8:447-453, 1986.

3. Pearson RD, Cox G, Jeronimo SM, Castracane J, Drew JS, Evans T, Alencar JE. Visceral leishmaniasis: a model for infection-induced cachexia. American Journal of Tropical Medicine 47 (suppl): 815, 1992.

4. Rombeau JL, Caldwell MD, Forlaw L, Guenter PA. Atlas of nutritional support techniques. Little, Brown and Co, Boston, 1989.

5. Waterlow JC. Classification and definition of protein-calorie malnutrition. British Medical Journal 2:566-569, 1972.

6. Witschi JC. Short-term dietary recall and recording methods. In: Willett W (ed) Nutritional epidemiology, Oxford, New York, p. 5268, 1989. 